\title{
GALACTIC WINDS IN ACTIVE GALAXIES
}

\author{
S. VEILLEUX, J. BLAND-HAWTHORN, G. CECIL \& P. SHOPBELL
}

University of Maryland

College Park, MD 20742 USA

Anglo-Australian Observatory

P.O. Box 296, Epping, NSW 2121 Australia

University of North Carolina

Chapel Hill, NC 27599 USA

\begin{abstract}
AND
California Institute of Technology

Pasadena, CA 91125 USA
\end{abstract}

\section{Introduction}

The effects of large-scale galactic winds in active galaxies may be farreaching. It has been suggested that the Hubble sequence can be understood in terms of a galaxy's greater ability to sustain winds with increasing bulge-to-disk ratio. The large-scale circulation of gas associated with these galactic winds might help explain the mass-metallicity relation between galaxies and the metallicity-radius relation within galaxies. Galactic winds probably contribute non-negligibly to the cosmic X-ray background and may be involved in the quasar absorption-line phenomenon. The cosmological implications of the wind phenomenon have been widely explored in the context of proto-galaxies and quasars. The extremely energetic galactic winds that were likely associated with galaxy formation almost certainly played a key role in heating and ionizing the intergalactic medium at high redshifts and may have created the seeds for the large-scale structure we see today. 


\section{On-Going Fabry-Perot Survey}

Using the Hawaii Imaging Fabry-Perot Interferometer (HIFI) at Mauna Kea and the TAURUS-2 Fabry-Perot system on the Anglo-Australian Telescope, we are acquiring Fabry-Perot data cubes on a complete sample of twenty nearby $(z<0.01)$ active galaxies (Seyferts and starbursts). So far, data have been obtained and analyzed for fifteen galaxies, and the results have been published for about half of these objects (cf. review by Veilleux et al. 1996). Our data reveal outflows which are highly complex, highly energetic ( $>10^{53} \mathrm{ergs}$ in most cases), and where the brightest emission often appears to be associated with strong shocks (e.g., Circinus: Veilleux \& Bland-Hawthorn 1997). Evidence for a loosely collimated wind which encompasses the radio jet is detected in several AGN (e.g., NGC 1068; Cecil et al. 1990). In starburst galaxies (e.g., M 82; Shopbell \& Bland-Hawthorn 1997), the outflowing material lies on the surface of bubbles or along the walls of funnel-shaped winds rapidly accelerating out of the galactic plane. The winds in both types of galaxies are generally lop-sided and tilted with respect to the polar axis of the host galaxy. Evidence for entrainment of disk material is seen in some objects (e.g., NGC 3079; Veilleux et al. 1994).

Our early results suggest that large-scale galactic winds can indeed have a strong impact on the thermal and chemical properties of some host galaxies and their surrounding environment. In no other galaxy is the impact of galactic winds more evident than in NGC 3079, a nearby edge-on spiral galaxy which is host to a spectacular kpc-scale bubble. Violent gas motions that range over $2,000 \mathrm{~km} \mathrm{~s}^{-1}$ are detected across the bubble and diametrically opposite on the other side of the nucleus. This is the most powerful example known of a windblown bubble, and an excellent laboratory to study wind dynamics. The dimensions and energies of the bubble imply that it is in the blowout phase and partially ruptured. A detailed dynamical analysis of this outflow indicates that the wind alone can contribute up to $5 t_{\text {outflow, } 8} \%$ of the total metal content of the host galaxy, where $t_{\text {outflow }, 8}$ is the outflow lifetime in units of $10^{8} \mathrm{yr}$ (Veilleux et al. 1994). We are currently analysing similar data on the other galaxies of our sample and plan to combine our results with those from future X-ray surveys to evaluate the wind's injection rate of hot, metal-enriched material into the host galaxy and surrounding intergalactic medium.

\section{References}

Cecil, G. N., Bland, J., \& Tully, B. T. 1990, Ap. J., 355, 70

Shopbell, P. L. \& Bland-Hawthorn, J. 1997, Ap. J., in press

Veilleux, S., \& Bland-Hawthorn, J. 1997, Ap. J., 479, L105

Veilleux, S., Cecil, G., Bland-Hawthorn, J. 1996, Scientific American, 274, 98

Veilleux, S., et al. 1994, Ap. J., 433, 48 